\title{
Dual Stenting Using Low-Profile LEO Baby Stents for the Endovascular Management of Challenging Intracranial Aneurysms
}

I. Akmangit, K. Aydin, S. Sencer, O.M. Topcuoglu, E.D. Topcuoglu, E. Daglioglu, M. Barburoglu, and A. Arat

\section{ABSTRACT}

BACKGROUND AND PURPOSE: Endovascular treatment of wide-neck, complex, and distally located cerebral aneurysms is a challenging issue. This study evaluated the safety and efficacy of dual stent placement by using a low-profile stent system (LEO Baby) for the treatment of challenging distal intracranial aneurysms.

MATERIALS AND METHODS: We retrospectively reviewed patients in whom at least 1 LEO Baby stent was used in the context of dual stent placement for the treatment of intracranial aneurysms. Patients who were treated with dual stent-assisted coil embolization and telescopic implantation of LEO Baby stents were included in the study. Clinical and angiographic findings, procedural data, and follow-up are reported.

RESULTS: Twelve patients were included in this study. Three patients presented with subarachnoid hemorrhage in the subacute-chronic phase, and the remaining patients had unruptured aneurysms. Nine patients were treated by using the dual stent-assisted coiling method. $\mathrm{X}$ - (nonintersecting), Y- (intersecting and reversible), T-, and parallel-stent configurations were performed for the dual stent-assisted coiling procedures. Three patients were treated by using telescopic stent placement for a flow diverter-like effect. The procedures were successful in all cases. Technical complications without a significant clinical adverse event developed in 2 patients. The 3- and 6-month control MRAs and DSAs demonstrated complete occlusion of the aneurysms in all patients except 1. All patients had good clinical outcomes on follow-up (mRS $\leq 1$ ).

CONCLUSIONS: The results of this small study showed the feasibility of dual stent placement by using low-profile LEO Baby stents to treat distally located complex intracranial aneurysms.

ABBREVIATION: AcomA = anterior communicating artery

C oiling of intracranial aneurysms is safe and effective, but endovascular treatment of wide-neck and anatomically complex aneurysms remains challenging. ${ }^{1}$ Self-expandable intracranial stents have been used in the past decade to achieve successful and durable coil embolizations of these aneurysms. ${ }^{2-4}$ The placement of a stent bridging the ostium of a wide-neck aneurysm

Received June 20, 2014; accepted after revision August 3.

From the Department of Radiology (I.A., E.D., A.A.), Ankara Numune Education and Research Hospital, Ankara, Turkey; Neuroradiology Division (K.A., S.S., M.B.), Department of Radiology, Istanbul Faculty of Medicine, Istanbul University, Istanbul, Turkey; Department of Radiology (I.A., O.M.T., A.A.), School of Medicine, Hacettepe University, Ankara, Turkey; and Department of Radiology (E.D.T.), School of Medicine, Ufuk University, Ankara, Turkey.

Please address correspondence to Anil Arat, MD, Department of Radiology, School of Medicine, Hacettepe University, Sihhiye, Ankara, Turkey; e-mail: anilarat@ hotmail.com

- Indicates open access to non-subscribers at www.ajnr.org

三 Indicates article with supplemental on-line table.

http://dx.doi.org/10.3174/ajnr.A4106 creates a scaffold, which prevents the protrusion or herniation of coils into the parent artery and results in denser coil packing. In addition to the mechanical effect, intracranial stents have hemodynamic and biologic effects. ${ }^{5-8}$ Stent deployment across the orifice of an aneurysm is thought to redirect blood flow from the sac of the aneurysm toward the distal parent artery and decrease the hemodynamic stress that contributes to thrombosis of the aneurysmal sac. ${ }^{9}$ Furthermore, stent-induced neointimal overgrowth leads to the healing of the neck of the aneurysm. ${ }^{10}$ On the basis of these effects, stent monotherapy has been proposed as an alternative strategy for the endovascular treatment of uncoilable and complex intracranial aneurysms. ${ }^{9-11}$ The telescopic placement of self-expandable intracranial stents can adequately divert the blood flow, especially for the treatment of blisterlike aneurysms. ${ }^{12}$

Recently, low-profile, self-expandable, braided intracranial stents (LEO Baby [Balt, Montmorency, France] and LVIS Jr. [MicroVention, Tustin, California]) have been available for the endovascular treatment of complex and/or distal aneurysms. ${ }^{13,14}$ 
These low-profile intracranial stents can be deployed into arteries with diameters of $<3.5 \mathrm{~mm}$ and delivered through microcatheters with an internal diameter of 0.0165 inches, which allows easier navigation in small-sized, delicate vessels.

A single stent may not suffice for the endovascular treatment of wide-neck and geometrically complex bifurcation aneurysms with involvement of 1 or both side branches. Endovascular treatment of these complex aneurysms often necessitates the implantation of 2 stents (ie, dual stent placement) in various configurations, such as Y-, X-, or parallel configurations. ${ }^{14-16}$

This retrospective study evaluated the safety and efficacy of dual stent placement by using a low-profile stent system (LEO Baby) for the treatment of challenging intracranial aneurysms.

\section{MATERIALS AND METHODS Patient Population}

We retrospectively reviewed interventional neuroradiology case records to identify patients in whom at least 1 LEO Baby stent was used in the context of dual stent placement for the treatment of intracranial aneurysms. Dual stent placement included Y-, X-, T-, or parallel configurations and the telescopic implantation of 2 low-profile stents without coiling (ie, telescopic stent monotherapy).

\section{Interventional Procedures}

All patients received $75 \mathrm{mg}$ of clopidogrel and $300 \mathrm{mg}$ of aspirin daily for at least 5 days before the procedure. In all cases, clopidogrel resistance was evaluated before the procedures (VerifyNow P2Y12 assay; Accumetrics, San Diego, California) to ensure a good response to clopidogrel. All endovascular procedures were performed with the patient under general anesthesia. Systemic anticoagulation was initiated immediately after the insertion of a femoral introducer sheath with a bolus dose of 5000 IU of IV heparin. The bolus dose was followed by a slow heparin infusion to maintain an activated clotting time of 2 times above the baseline value. A $6 \mathrm{~F}$ guiding sheath was placed in the common carotid artery or the subclavian artery at the beginning of the procedure, and a distal-access catheter (Fargomax [Balt] or Neuron 070 delivery catheter [Penumbra, Alameda, California]) was navigated through the sheath into the intracranial internal carotid artery or to the V2 segment of the vertebral artery.

Stent-Assisted Coiling. Stent-assisted coil embolization of the aneurysms was performed by using the jailing technique. Aneurysm sacs were catheterized by using an Excelsior SL-10 (Stryker Neurovascular, Fremont, California) or Headway 17 microcatheter (MicroVention/Terumo) with a soft-tip 0.014-inch microguidewire before stent deployment. A second microcatheter (Vasco 21 [Balt] for the LEO stent, Prowler Select Plus [Codman \& Shurtleff Neurovascular, Raynham, Massachusetts] for the Enterprise stent [Codman \& Shurtleff], Excelsior SL-10 [Stryker Neurovascular] or Headway 17 for LEO Baby stents) was navigated across the aneurysm. For Y- and T-stents, including the temporary $\mathrm{Y}$, the first stent was deployed and the microcatheter was navigated through the stent over the delivery wire. The delivery wire was exchanged with a microguidewire, the second side branch was accessed by using the same microcatheter, and the second stent was deployed (for the reversible $\mathrm{Y}$, it was partially deployed and retrieved later). For $\mathrm{X}$ - and parallel-stent place- ment, 2 microcatheters (Excelsior SL-10 or Headway 17) were placed from the ipsilateral A1 to the ipsilateral (parallel type) or contralateral A2 segments (X type). An exchange maneuver was performed after the deployment of the first stent to replace the larger microcatheters with an Excelsior SL-10 or Headway 17 when stents other than the LEO Baby were used as the first stent.

Coiling was performed with bare platinum detachable coils after the stents were deployed across the neck of the aneurysm. The aneurysms were coil-embolized until complete occlusion was achieved or no further coils could be safely deployed within the aneurysm sac.

Telescopic Dual Stent Placement with or without Coiling. We preferred to perform a dual stent monotherapy by using the telescopic implantation of 2 low-profile LEO Baby stents to achieve flow diversion. The microcatheter was navigated across the aneurysm neck during this procedure. Two LEO Baby stents were deployed telescopically within each other at the neck of aneurysms by using the same microcatheter. In 2 patients with dissecting aneurysms, stent monotherapy was performed; in 1 patient, subtotal coiling was performed.

\section{Follow-Up}

Immediate postprocedural angiograms were obtained at the end of the embolization procedures. Patients were asked to return at 3 months for MR angiography and at 6 months for a digital subtraction angiography follow-up. Patients' neurologic statuses were evaluated at the time of follow-up imaging by using the modified Rankin Scale. Postprocedural dual antiplatelet treatment, including $75 \mathrm{mg}$ /day of clopidogrel and $300 \mathrm{mg} /$ day of aspirin, was continued for 6 months. Then, dual antiplatelet therapy was switched to $300 \mathrm{mg} /$ day of aspirin monotherapy.

\section{RESULTS}

Twelve patients, 9 women and 3 men, were included in the study. The mean age was 55.2 years (range, $30-69$ years). Three patients presented with subarachnoid hemorrhage in the subacute (1-3 weeks) phase, and the remaining patients had unruptured aneurysms. Five of the 12 aneurysms were located at the anterior communicating artery (AcomA), 2 aneurysms were located at the basilar artery, 1 aneurysm was located at the posterior inferior cerebellar artery, 1 aneurysm was located at the A1 segment of the anterior cerebral artery, and 3 aneurysms were located at the MCA bifurcation. The mean aneurysm size was $9.8 \mathrm{~mm}$ (range, 3-22 $\mathrm{mm}$ ). One patient had a previous coiling (patient 9), and another patient had a previous surgical clipping (patient 6) of the target aneurysm. Data on patient characteristics and procedures are summarized in detail in the On-line Table.

The dual stent-assisted coiling procedure was performed in 9 patients. Y-stent placement was performed in 5 patients (Fig 1), 1 of whom underwent temporary Y-stent placement. T- and nonintersecting X- (Fig 2) and parallel-stent placement were performed in 4 additional patients. The patient whose AcomA aneurysm (patient 4) was treated by using the nonintersecting $\mathrm{X}$-stent-assisted coiling method also had 2 additional aneurysms located on the supraclinoid segment of left internal carotid artery. A flow-diverter stent (Silk; Balt) was implanted across the neck of these ICA aneurysms in the same session with X-stent placement 

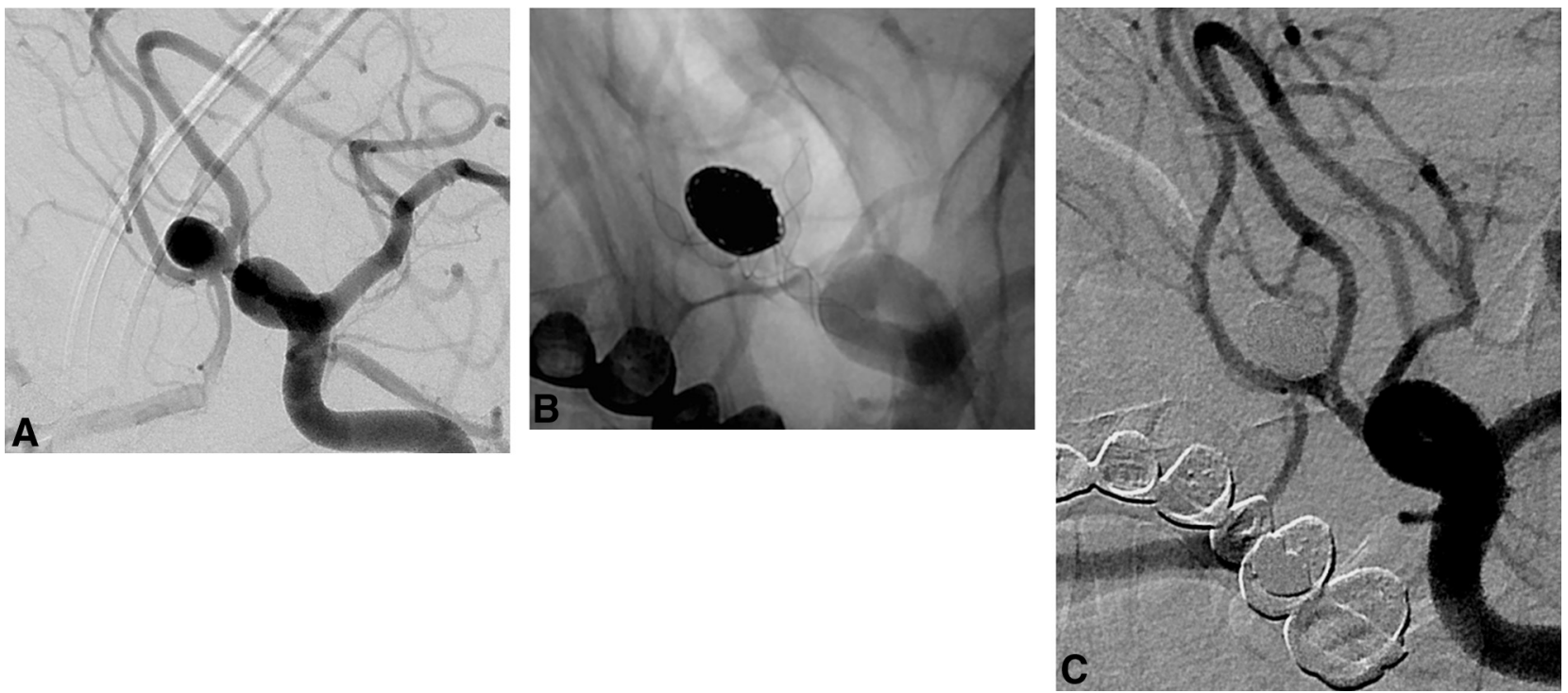

FIG 1. Procedural and follow-up DSAs of patient 3. A, DSA in the oblique-Waters projection revealing an 8-mm AcomA aneurysm with a wide neck extending to both $A 2$ segments and a separate baby aneurysm at the left A1-A2 junction. $B$, The postprocedural native image from DSA shows that the aneurysm was well-embolized by stent-assisted coiling by using 2 LEO Baby stents in a Y-configuration. C, DSA in the 6-month angiogram shows no residual aneurysm and patent stents.
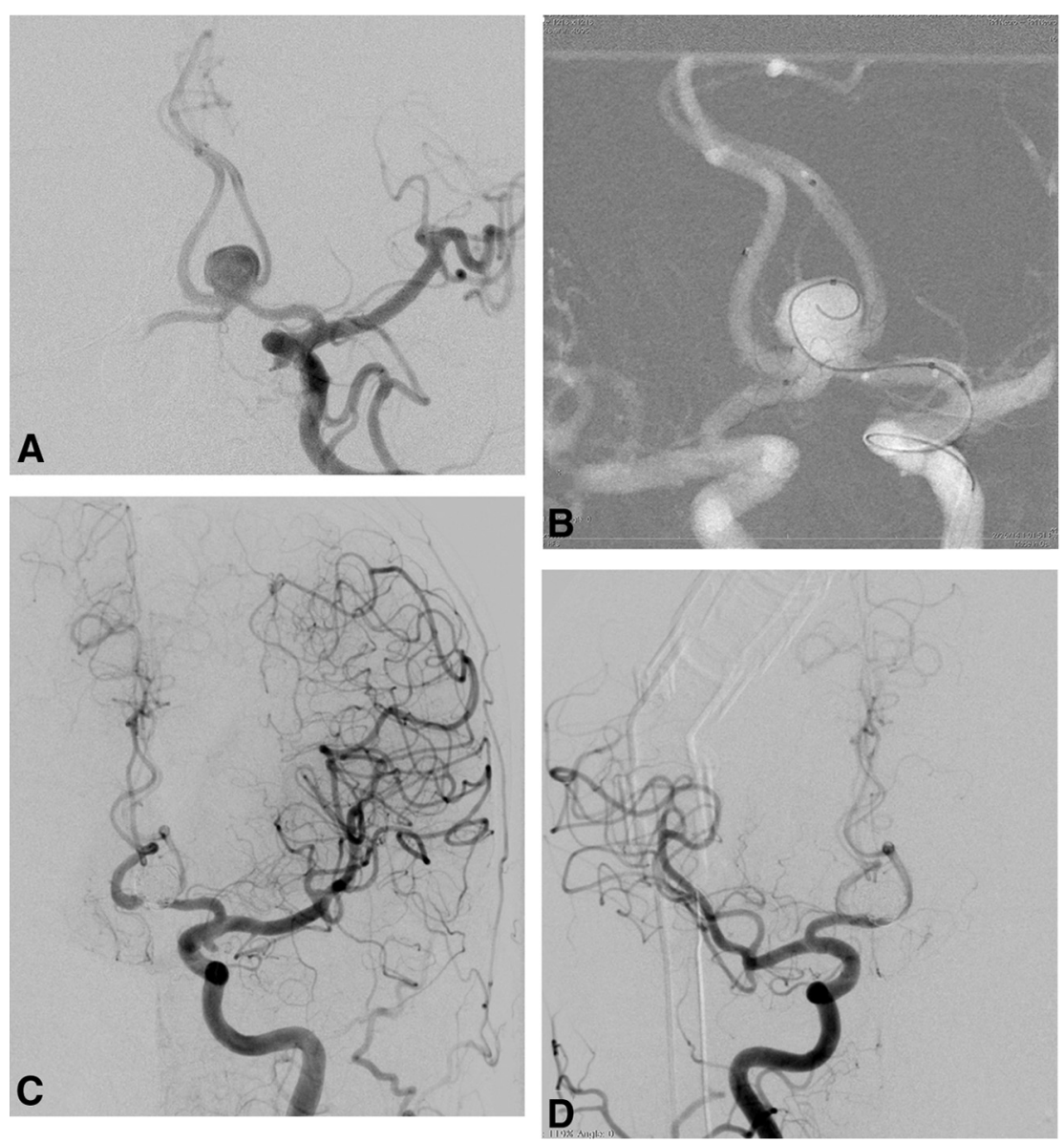

FIG 2. Procedural DSA images of patient 4. A, A 9-mm AcomA aneurysm with a wide neck extending to both $A 2$ segments. $B$, A roadmap capture in the Waters projection obtained after bilateral simultaneous internal carotid artery injections. It shows nonintersecting X-stent placement and the jailed microcatheter within the aneurysm sac. $C$ and $D$, The postprocedural DSA images after both left internal carotid artery injections show coiling by using 2 LEO Baby stents in an X-configuration.
(Fig 2). In 1 patient, telescopic stent placement following the partial coiling was performed in an effort to achieve flow diversion because stent-assisted coiling with a single stent was suboptimal due to recurrent microcatheter kickback from the shallow aneurysm (patient 11). Including patient 11 , telescopic stent placement was performed in 3 patients (Fig 3). Only LEO Baby stents were used for dual stent placement in 8 patients. Enterprise and LEO stents were used as the first stent in 4 cases followed by the placement of LEO Baby stents. LEO Baby stents were successfully deployed in all patients except patient 7 , in whom migration of the LEO Baby occurred during deployment. Immediate postprocedural DSA images demonstrated a complete or near-complete occlusion in 9 aneurysms that were treated by using dual stent-assisted coiling techniques (Fig 4). The immediate postprocedural DSA of patients who underwent telescopic stent placement, including patient 11 , revealed a complete occlusion of the aneurysm in 1 patient and a stagnated filling of the neck in the 2 other patients.

Two technical complications without significant clinical adverse events were noted in this series. Stent placement with a reversed T-stent was attempted for the treatment of an MCA 

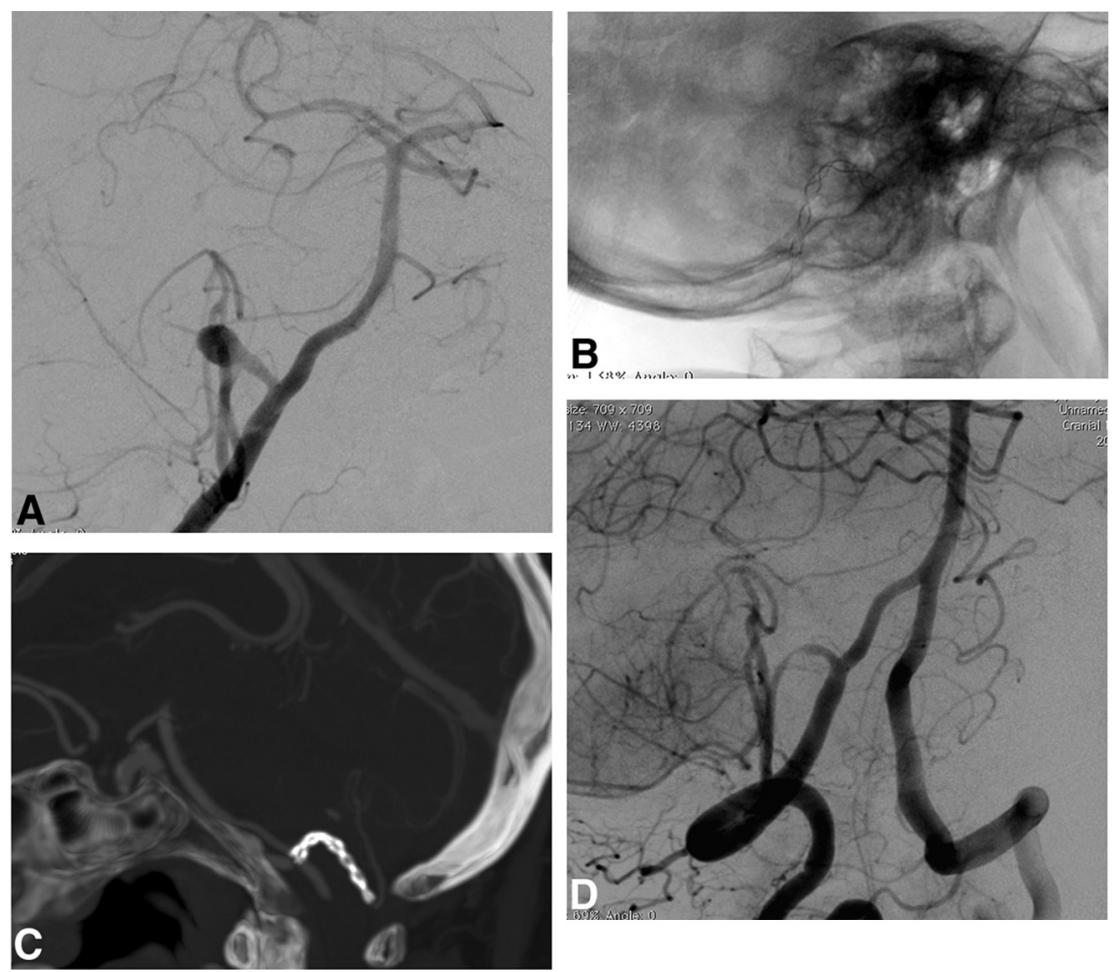

FIG 3. DSA and CT angiography images of patient 8 . A, DSA image after the vertebral artery injection shows a 9-mm dissecting aneurysm located at the origin of the right posterior inferior cerebellar artery. $B$, Unsubtracted DSA image obtained during endovascular treatment shows 2 telescopically implanted LEO Baby stents. C, Three-month follow-up CT angiogram shows the patent stents and no filling of the aneurysm. $D$, Six-month follow-up DSA image in the oblique position reveals no residual aneurysm filling and some neointimal growth within patent stents.

bifurcation aneurysm in patient 7 . The first stent was deployed precisely during the procedure; the proximal tip of the stent ended exactly at the start of the superior MCA trunk, which originated from the aneurysm. However, we realized that the deployment system was stuck when we attempted to release the stent at the desired location, and the stent could not be released from the delivery wire despite being fully deployed. The stent was released from the delivery wire and migrated into the aneurysm sac following a gentle backward and forward manipulation of the wire, which was fixed to the stent. The aneurysm sac was catheterized by using the same microcatheter that was used for stent deployment, and the migrated stent was immobilized through the placement of several coils into the aneurysm sac. Two more stents in a Y-configuration were consecutively placed into the lower and upper MCA trunks to complete the coil embolization. Coiling was finalized in the usual manner without sequelae.

Temporary Y-stent placement was planned in 1 patient (patient 1) who had an MCA bifurcation aneurysm due to the high risk of a kickback of the microcatheter from the shallow aneurysm sac during coiling. A LEO Baby stent was deployed in the superior trunk of the MCA. A second LEO Baby stent was released in the inferior trunk for approximately $60 \%$ of the length of the stent with the intention of pulling it back after coiling. An instantaneous extravasation from the aneurysm was noted during the procedure, and coiling was continued with the assistance of both stents. However, the microcatheter kicked back from the aneurysm sac. The partially opened second stent was withdrawn in response, and access to the aneurysm sac was attained with the microcatheter to complete the coiling. The patient awoke without neurologic deficits. Cerebral CT revealed that the contrast extravasation was confined to the Sylvian fissure.

Six-month follow up DSAs were available in 8 of the 12 patients. They demonstrated a stable occlusion of the aneurysms in all 8 patients. One patient (patient 7) had a 3-month follow-up DSA, which demonstrated the recurrence of the treated aneurysm. Two patients had 3-month follow-up MRAs that revealed a complete occlusion of the aneurysms in both. In 1 patient (patient 6), follow-up DSA at 6 months showed the occlusion of both A1 segments with robust retrograde opacification of the distal anterior cerebral arteries from the leptomeningeal collaterals. This patient did not reliably adhere to her antiplatelet medication regimen after discharge. The finding was incidental, and the patient did not have any neurologic signs or symptoms. We had not performed any follow-up imaging examination in 1 patient (patient 11) at the time of submission of this article. Neurologic control examinations at follow-ups revealed good clinical outcomes in all patients (mRS scores ranged between 0 and 1).

\section{DISCUSSION}

Several endovascular techniques have been defined for the treatment of wide-neck intracranial aneurysms. Balloon-assisted coiling, stent-assisted coiling, dual or telescopic stent placement, and flow diversion by using dedicated flow diverters are the most commonly used methods for the endovascular treatment of wideneck aneurysms. Balloon-assisted coiling is a relatively safe method, but it is associated with retreatment in the long term. ${ }^{17,18}$ Stent-assisted coiling addresses both the defective wall segment of the parent artery and the aneurysm sac. ${ }^{19,20}$ Stents may enable endovascular treatment in otherwise uncoilable aneurysms by providing a scaffold during coiling. Moreover, stents decrease aneurysm recurrence rates by altering hemodynamic effects and stimulating healing reactions. ${ }^{19,20}$

Dual stent placement may be required for the endovascular treatment of some aneurysms, especially aneurysms located at the bifurcations and AcomA. ${ }^{20-23}$ Chow et al ${ }^{16}$ first defined stentassisted coiling in the Y-configuration in 2004. Menendez and Harrigan ${ }^{15}$ described X-configuration stent placement, and dual stent placement is now widely used for the treatment of wide-neck complex aneurysms. ${ }^{20-23}$ Recently, several successful series of Yor X-stent-assisted coiling by using low-profile stents have been 



FIG 4. Procedural DSA images of patient 10. A, DSA image after the injection of left ICA reveals a fusiform 7-mm MCA bifurcation aneurysm extending to both MCA trunks. B, Roadmap-capture image in an oblique working projection shows the implantation of 2 LEO Baby stents in a T-configuration. C, The postprocedural DSA image in the working projection demonstrates the embolized aneurysm sac and the small residual "dog-ear" fillings at the origin of both trunks.

published. ${ }^{13,24}$ Möhlenbruch et $\mathrm{al}^{24}$ reported 22 patients treated with LVIS Jr. stents, 9 of whom underwent dual stent placement. In another study, 11 of 34 aneurysms treated with LVIS Jr. stents underwent dual stent placement. ${ }^{13}$ However, only the first report provides details about the outcomes of dual stent placement by using low-profile stents, and this study suggested that these stents are safe to use in dual stent-assisted coiling. To our knowledge, there are no previous studies on dual stent placement by using the low-profile LEO Baby stent used in our series. We treated complex/distal intracranial aneurysms by using dual stent placement with the LEO Baby stent. Our case series demonstrates that favorable immediate and early follow-up angiographic results can be attained with dual stent placement by using low-profile intracranial stents that are deployed in various configurations, including telescopic stent placement for the treatment of wide-neck, complex aneurysms. We found that various dual stent placement configurations, such as Y-, nonintersecting X-, T-, and parallel-stent placement, could be successfully performed by using double LEO Baby stents or a single stent in combination with larger intracranial stents. To the best of our knowledge, there is only 1 case report of the use of LEO Baby stents for dual stent placement (nonintersecting X-stent placement) in the literature, ${ }^{14}$ and there are no reports on intersecting (ie, Y or reversible $\mathrm{Y}$ ) or tangential dual stent placement (ie, T).

LEO Baby stents are self-expandable, low-profile stents composed of braided mesh nitinol wires. Two platinum wires enable radiographic visualization of the stent. LEO Baby stents, similar to the LVIS Jr. stents, exhibit several advantages over laser-cut stents. First, LEO Baby stents have a sliding-strut design, and this hybrid design allows better wall apposition and scaffolding compared with open and closed cells. Second, LEO Baby stents are resheathable or re-positionable up to approximately $95 \%$ of their length. ${ }^{14}$ This feature is a major advantage over nonretrievable open-cell stents and Enterprise stents, which can be retrieved up to $70 \%$ of their length, and the LVIS Jr. stent, which can be withdrawn until it is $80 \%$ released. ${ }^{24,25}$ Finally, the LEO Baby stent fits inside microcatheters with luminal diameters of 0.0165 inches; therefore, these stents may exhibit improved navigation within arteries or through already-deployed stents. The struts of LEO Baby stents are not fixed (ie, the struts can move over each other); this feature makes room for the second stent and decreases the constraint on the second stent at the level of the stent intersection. Catheterization of the side branch through the stent is possible for the same reason. Similar to LEO stents, 2 radiopaque platinum makers of the LEO Baby stents produce linear artifacts, which would imitate an in-stent stenosis on the reconstructed MRA images. ${ }^{26}$ However, the LEO Baby stents do not produce any significant artifacts outside the stent, and evaluation of residual or recurrent filling of the aneurysm is straightforward. Thus, we used contrast-enhanced MRA for 3-month follow-up imaging of our patients.

Several differences exist between the LVIS jr. stent and the LEO Baby stent. The major difference is the cell size. The LEO Baby stent has a cell size of approximately $0.9 \mathrm{~mm}$, which is significantly smaller than that in the LVIS Jr. stent $(1.5 \mathrm{~mm}) .{ }^{24} \mathrm{We}$ can cross the LEO Baby stent with small microcatheters (1.7F internal diameter) for Y-stentings, despite the very small cell size. However, every effort should be made to jail the microcatheter 
before stent placement and to stent the harder-to-access branch first. Otherwise, the angle of entry through the attenuated stent mesh may not allow safe intra-aneurysmal or side-branch access. One advantage of this attenuated mesh is that the telescopic configuration may enhance healing by acting as a miniature flow diverter, as demonstrated in 2 of our telescopic configurations. Additionally, LEO Baby stent placement is intended for arteries as small as $1.5 \mathrm{~mm}$ (versus $2.0 \mathrm{~mm}$ ), and it has been used in smaller arteries. $^{14,27}$ The second difference is the presence of shorter flared ends in the LEO Baby stent, which potentially eases re-entry to the stent for dual stent placement once it is deployed. Finally, the LEO Baby stent is composed of 16 wires versus the 12 wires in the LVIS Jr., which increase its radial force. ${ }^{14}$ This difference may have prevented incomplete stent opening in the 20 stents deployed in this series. This adverse event occurs at a rate of $6 \%-7 \%$ with the LVIS Jr. ${ }^{24,25}$

One technical complication, stent migration into the aneurysm after deployment, developed in 1 of our patients, but this complication did not result in any clinical consequences. Stent migration during deployment was reported by using other types of open-cell or closed-cell stents, but migration may be more of a concern with low-profile braided stents because they have a lower radial force. Not surprising, only 1 stent migration was reported in the 22 patients treated by Möhlenbruch et al. ${ }^{24}$ The underlying cause for this technical complication was manipulation of the delivery wire, which was stuck in the stent. This adverse event, to the best our knowledge, has not been reported previously by using low-profile stents. Another complication was occlusion of the parent artery without any clinical deficit at the 6-month followup. This patient did not adhere to the antiplatelet medication regimen after discharge. An early switch from dual antiplatelet therapy to aspirin monotherapy increases the risk of delayed thromboembolic complications in patients treated by stent-assisted coiling. ${ }^{28}$ When we considered the distal locations of the aneurysms and relatively small sizes of the parent arteries in our cases, we continued the dual antiplatelet therapy for 6 months after the procedures. The newer versions of the LEO stents, including LEO Baby stents, undergo a new surface-modification procedure $^{29}$ to decrease thromboembolic events, but a higher risk of thromboembolic events is expected with the use of these stents in smaller stented arteries. Möhlenbruch et $\mathrm{al}^{24}$ reported that the risk of transient thromboembolic events was 14\%, which is higher than normal for stents placed in large intracranial arteries. Intuitively, we anticipate a considerable thromboembolic risk for dual stent placement in small-caliber arteries and advocate heavy reliance on point-of-care testing and adenosine diphosphate/thrombin receptor agonist peptide values from the hospital laboratory to exclude patients with clopidogrel hyposensitivity.

Temporary stent placement with laser-cut intracranial stents has been described recently for ruptured aneurysms. ${ }^{30}$ Our series demonstrated that this technique could be applied by using lowprofile braided stents. Furthermore, this technique can also be used in unruptured aneurysms when there is a high risk of rupture or catheter kickback during dual stent placement, as shown in our series.

Our series reported successful results of telescopic dual-stent monotherapy for the treatment of 2 distally located, complex an- eurysms. To the best of our knowledge, these patients are the first to have reports of telescopic low-profile stent implantation as stent monotherapy for the treatment of intracranial aneurysms. The aneurysms in these patients were distally located, partially thrombosed, and wide-neck. In another patient, we performed telescopic stent placement following an incomplete stent-assisted coiling to achieve complete occlusion. The placement of 2 LEO Baby stents inside one another telescopically may act as a flow diverter for distally located and/or dissecting aneurysms due to their attenuated mesh and higher wall coverage. The telescopic placement of low-profile braided stents may be an alternative to dedicated flow diverters and larger stents in parent arteries of $<2$ $\mathrm{mm}$ because these devices pass through larger and stiffer microcatheters with limited capacity during the catheterization of very distal aneurysms.

\section{CONCLUSIONS}

The short-term follow-up results of dual stent placement by using low-profile intracranial stents for the treatment of complex intracranial aneurysms in this small series are encouraging. This small study showed the feasibility of dual stent placement by using lowprofile stents to treat distally located complex intracranial aneurysms. Moreover, the techniques were effective and were not associated with clinically significant adverse events.

\section{REFERENCES}

1. Molyneux A, Kerr R, Stratton I, et al. International Subarachnoid Aneurysm Trial (ISAT) of neurosurgical clipping versus endovascular coiling in 2143 patients with ruptured intracranial aneurysms: a randomised trial. Lancet 2002;360:1267-74

2. Wanke I, Doerfler A, Schoch B, et al. Treatment of wide-necked intracranial aneurysms with a self-expanding stent system: initial clinical experience. AJNR Am J Neuroradiol 2003;24:1192-99

3. Akpek S, Arat A, Morsi H, et al. Self-expandable stent-assisted coiling of wide-necked intracranial aneurysms: a single-center experience. AJNR Am J Neuroradiol 2005;26:1223-31

4. Lubicz B, Leclerc X, Levivier M, et al. Retractable self-expandable stent for endovascular treatment of wide-necked intracranial aneurysms: preliminary experience. Neurosurgery 2006;58:451-57

5. Wanke I, Forsting M. Stents for intracranial wide-necked aneurysms: more than mechanical protection. Neuroradiology 2008;50:991-98

6. Tateshima ST, Hakata Y, Tanoue SY, et al. Alteration of intraaneurysmal hemodynamics by placement of a self-expandable stent: laboratory investigation. J Neurosurg 2009;111:22-27

7. Shobayashi Y, Tateshima S, Kakizaki R, et al. Intra-aneurysmal hemodynamic alterations by a self-expandable intracranial stent and flow diversion stent: high intra-aneurysmal pressure remains regardless of flow velocity reduction. J Neurointerv Surg 2013;5(suppl 3): iii $38-42$

8. Kono K, Terada T. Hemodynamics of $\mathbf{8}$ different configurations of stenting for bifurcation aneurysms. AJNR Am J Neuroradiol 2013; 34:1980-86

9. Fiorella D, Albuquerque FC, Deshmukh VR, et al. Endovascular reconstruction with the Neuroform stent as monotherapy for the treatment of uncoilable intradural pseudoaneurysms. Neurosurgery 2006;59:291-99; discussion 299-300

10. Li C, Li Y, Jiang C, et al. Stent alone treatment for dissections and dissecting aneurysms involving the basilar artery. J Neurointerv Surg 2015;7:50-55

11. Bulsara KR, Kuzmik GA, Hebert R, et al. Stenting as monotherapy for uncoilable intracranial aneurysms. Neurosurgery 2013;73(1 suppl operative):ons $80-85$

12. Walsh KM, Moskowitz SI, Hui FK, et al. Multiple overlapping stents 
as monotherapy in the treatment of 'blister' pseudoaneurysms arising from the supraclinoid internal carotid artery: a single institution series and review of the literature. J Neurointerv Surg 2014; 6:184-94

13. Behme D, Weber A, Kowoll A, et al. Low-profile Visualized Intraluminal Support device (LVIS Jr) as a novel tool in the treatment of wide-necked intracranial aneurysms: initial experience in 32 cases. J Neurointerv Surg 2014 Apr 10. [Epub ahead of print]

14. Cohen JE, Melamed I, Itshayek E. X-microstenting and transmesh coiling in the management of wide-necked tent-like anterior communicating artery aneurysms. J Clin Neurosci 2014;21:664-67

15. Menendez JY, Harrigan MR. X-configuration stent-assisted coiling. World Neurosurg 2010;74:143-44

16. Chow MM, Woo HH, Masaryk TJ, et al. A novel endovascular treatment of a wide-necked basilar apex aneurysm by using a Y-configuration, double-stent technique. AJNR Am J Neuroradiol 2004; 25:509-12

17. Shapiro M, Babb J, Becske T, et al. Safety and efficacy of adjunctive balloon remodeling during endovascular treatment of intracranial aneurysms: a literature review. AJNR Am J Neuroradiol 2008; 29:1777-81

18. Chalouhi N, Starke RM, Koltz MT, et al. Stent-assisted coiling versus balloon remodeling of wide-neck aneurysms: comparison of angiographic outcomes. AJNR Am J Neuroradiol 2013;34:1987-92

19. Raymond J, Darsaut TE, Bing F, et al. Stent-assisted coiling of bifurcation aneurysms may improve endovascular treatment: a critical evaluation in an experimental model. AJNR Am J Neuroradiol 2013;34:570-76

20. Thorell WE, Chow MM, Woo HH, et al. Y-configured dual intracranial stent-assisted coil embolization for the treatment of widenecked basilar tip aneurysms. Neurosurgery 2005;50:1035-40

21. Cho YD, Park SW, Lee JY, et al. Nonoverlapping Y-configuration stenting technique with dual closed-cell stents in wide-neck basilar tip aneurysms. Neurosurgery 2012;70:244-49

22. Johnson AK, Heiferman DM, Lopes DK. Stent-assisted embolization of $\mathbf{1 0 0}$ middle cerebral artery aneurysms. J Neurosurg 2013; 118:950-55

23. Akgul E, Aksungur E, Balli T, et al. Y-stent assisted coil embolization of wide-neck intracranial aneurysms. Interv Neuroradiol 2011; $17: 36-48$

24. Möhlenbruch M, Herweh C, Behrens L, et al. The LVIS Jr. microstent to assist coil embolization of wide-neck intracranial aneurysms: clinical study to assess safety and efficacy. Neuroradiology 2014;56:389-95

25. Poncyljusz W, Biliński P, Safranow K, et al. The LVIS/LVIS Jr. stents in the treatment of wide-neck intracranial aneurysms: multicentre registry. J Neurointerv Surg 2014 May 14. [Epub ahead of print]

26. Lövblad KO, Yilmaz $\mathrm{H}$, Chouiter $\mathrm{A}$, et al. Intracranial aneurysm stenting: follow-up with MR angiography. J Magn Reson Imaging 2006;24:418-22

27. LEO + \& LEO + Baby. Version DC015GB 2013/04 [brochure]. Mayabee Design Studio. Seoul: Balt Extrusion, 2013

28. Hwang G, Kim JG, Song KS, et al. Delayed ischemic stroke after stent-assisted coil placement in cerebral aneurysm: characteristics and optimal duration of preventative dual antiplatelet therapy. $R a-$ diology 2014;273:194-201

29. LEO +, next generation self-expanding intracranial stent. Version DC015GB 2010/03 [brochure]. Mayabee Design Studio. Seoul: Balt Extrusion, 2010

30. Signorelli F, Gory B, Turjman F. Temporary Solitaire stent-assisted coiling: a technique for the treatment of acutely ruptured wideneck intracranial aneurysms. AJNR Am J Neuroradiol 2014;35: $984-88$ 